\title{
Reproducibility of Heart Rate Variability Characteristics Measured on Random 10-second ECG using Joint Symbolic Dynamics
}

\author{
Muammar M Kabir ${ }^{1}$, Golriz Sedaghat ${ }^{1,2}$, Jason Thomas ${ }^{1}$, Larisa G Tereshchenko ${ }^{1}$ \\ ${ }^{1}$ Knight Cardiovascular Institute, Oregon Health and Science University, Portland, OR, USA \\ ${ }^{2}$ Portland State University, Portland, OR, USA
}

\begin{abstract}
In this paper we employed joint symbolic dynamics (JSD) approach to study reproducibility of heart rate variability characteristics measured on 2 randomly selected 10-second segments within 3-minute resting orthogonal ECG in 170 healthy participants. First, the ECG R-peaks were detected using parabolic fitting. Second, the respiratory signal was derived from orthogonal ECG X-lead using QRS slopes. Third, time series of $R$ - $R$ intervals and respiratory phases (calculated using Hilbert transform), were transformed into tertiary symbol vectors based on their successive changes and words of length ' 3 ' were formed. Bland-Altman analysis was used to assess the agreement between measured logtransformed JSD characteristics of HRV, and their reproducibility. Traditional HRV measures such as RR' interval changes showed a very high reproducibility. However, agreement between two 10-second JSD indices of $H R V$ was low. Interestingly, a significant decrease in low-high alterations of HRV dynamics measured using JSD was observed when respiratory phase transition intervals were excluded (10s: $4.7 \pm 9.4$ vs. $24.8 \pm 21.0 \%$, $p<0.0001$; 3min: $9.8 \pm 8.1$ vs. $24.8 \pm 12.3 \%, p<0.0001$ ).
\end{abstract}

\section{Introduction}

The study of heart rate variability (HRV) provides clinically relevant information about autonomic control [1]. Compared to conventional signal-processing approaches which are inadequate for characterizing complex system dynamics, joint symbolic dynamics (JSD) has been shown to be an effective technique to provide enhanced information by employing a coarsegraining procedure to preserve the robust properties of the system's complex dynamics [2,3]. Symbolic dynamics has been widely used to study HRV dynamics and is suggested to provide improved performance for the analysis of respiratory data and cardiorespiratory interaction $[3,4]$.

Standard short-term HRV analysis traditionally requires 5-minutes of continuous ECG recording. Recently, it has been shown that the quantitative estimation of HRV parameters is not significantly affected by shortening the duration of analyzed ECG to 30-60 second segments [5]. In clinical practice, 10-second ECG recording is routinely available and therefore could provide clinical studies with the opportunity to investigate clinical utility of HRV characteristics for risk assessment in patients. However, reproducibility of HRV indices using JSD has never been studied.

Increased repetitive low-high alterations between intervals of major near-field deflections on right ventricular intracardiac electrogram analyzed using tertiary symbolization for JSD has been shown to be associated with mortality [6]. Respiratory sinus arrhythmia is modulated by vagal tone, and is characterized by heart rate accelerations/decelerations that occur in phase with inhalation and exhalation during respiratory cycle. Consequently, low-high alterations in HRV symbolic dynamics may occur during the inhalation-exhalation phase transition. The aim of this study was to evaluate reproducibility and to determine the effect of respiratory phase transition on HRV characteristics measured using JSD. We hypothesize that HRV symbolic characteristics are affected by respiratory phase transitions.

\section{Methods}

The study conformed to principles outlined in the Declaration of Helsinki and was approved by the local ethics committee. Each participant provided written informed consent.

\subsection{Subjects}

This study population was composed of 170 healthy participants of the prospective cohort Intercity Digital Electrogram Alliance (IDEAL) study, provided by the NIH-funded The Telemetric and Holter ECG Warehouse initiative [7]. Mean age was $38.1 \pm 15.2$ years, $50 \%$ were male and $94 \%$ were white. The participants had normal 
echocardiogram, physical examination and stress test, 12lead ECG in sinus rhythm, and no history of any chronic illness.

\subsection{Data recording}

High resolution $(1000 \mathrm{~Hz})$ modified $\left(5^{\text {th }}\right.$ intercostal space) Frank orthogonal ECG (XYZ) was recorded by the SpaceLab-Burdick digital Holter recorder (SpaceLabBurdick, Inc. Deerfield, WI) at rest in the supine position for at least 3 minutes.

\subsection{Processing of ECG, derivation of respiratory signal and $\mathrm{HRV}$ analysis}

Two non-adjacent 10-second ECG segments were selected from all ECG recordings. Custom-written computer software developed in MATLAB ${ }^{\circledR}$ was used for the detection of QRS onsets and offsets, and R-peaks on orthogonal X-lead. All ECG segments were visually scanned for presence of noise distortion or irregular beats.

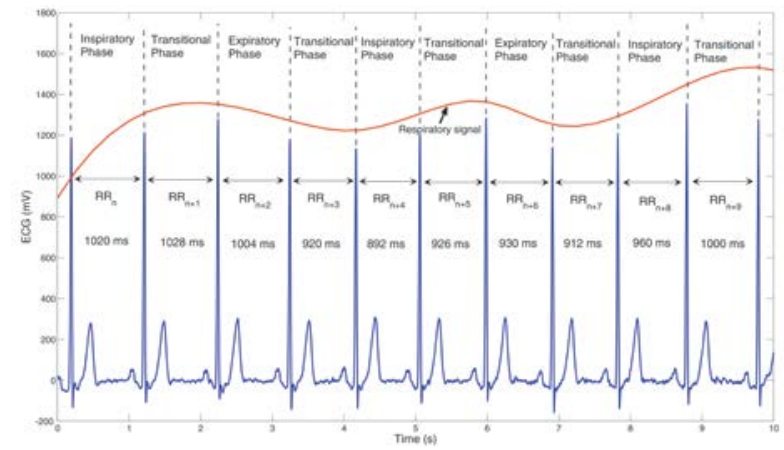

Figure 1. Illustration of changes in RR' intervals and changes in phases of ECG derived respiratory signal.

Respiratory signal was derived from the orthogonal Xlead using a recent method presented by Lazaro et al. [8]. For each QRS complex, the upward slope and the downward slope of the R-wave were measured. From the sample point associated with the maximum slope, a straight line of $8 \mathrm{~ms}$ interval was fitted to each side of the $\mathrm{R}$-wave. The slopes of the lines were the required QRS slopes. By adding the series of the QRS slopes an unevenly sampled respiratory signal was obtained. This signal was then interpolated at $4 \mathrm{~Hz}$ using cubic-splines interpolation technique to obtain an evenly sampled ECGderived respiratory signal (Fig.1). The Hilbert transform was used to calculate the phases of the respiratory signal.

For the analysis of traditional HRV, the following indices were calculated: meanNN (mean value of sinus RR' time series), sdNN (standard deviation of sinus RR' time series), rMSSD (square root of the mean squared differences of successive RR' intervals) and PPA_S1S2 (ratio of the standard deviation of the short-term RR' interval variability to the standard deviation of the longterm RR' interval variability in the Poincare plot).

\subsection{Joint symbolic dynamics}

From the vectors of R-R time series and respiratory phases, RP (taken at the instants of R-peaks), we established two symbolic sequences, $s^{\mathrm{RR}}$ and $s^{\mathrm{RP}}$, using the transformation rules below, respectively. They are based on the differences between successive R-R intervals (an increase in RR' was represented by “+1”, decrease by " 1 " and no change by " 0 ") and R-instant respiratory phases (inspiratory phase was represented by " +1 ", expiratory phase by " -1 " and transition from inspiratory to expiratory phase and vice versa by “ 0 ”), as described previously [3].

$$
\begin{aligned}
& S_{i}^{\mathrm{RR}}=\left\{\begin{array}{l}
-1 \text { if } \mathrm{RR}_{i+1}-\mathrm{RR}_{i}<\mathrm{Th} \\
0 \text { if } \mathrm{RR}_{i+1}-\mathrm{RR}_{i}=\mathrm{Th} \\
1 \text { if } \mathrm{RR}_{i+1}-\mathrm{RR}_{i}>\mathrm{Th}
\end{array}\right. \\
& S_{i}^{\mathrm{RP}}=\left\{\begin{array}{l}
-1 \text { if } \mathrm{RP}_{i+1}>0 \& \mathrm{RP}_{i}>0 \\
0 \text { if }\left(\mathrm{RP}_{i+1}<0 \& \mathrm{RP}_{i}>0\right) \text { or }\left(\mathrm{RP}_{i+1}>0 \& \mathrm{RP}_{i}<0\right) \\
1 \text { if } \mathrm{RP}_{i+1}<0 \& \mathrm{RP}_{i}<0
\end{array}\right.
\end{aligned}
$$

Here, Th represents a defined threshold value which allows distinguishing high beat-to-beat variability from small changes that might be within the noise floor. For this study $T h=2$ was used. Subsequently, series of words, $w^{\mathrm{RR}}$ and $w^{\mathrm{RP}}$ of length three were constructed. Consequently, eight different word types were obtained for each vector [3].

By evaluating the probability distribution of the $27\left(3^{3}\right)$ possible word pattern types, the following indices were calculated by grouping individual word patterns into various families [9]: R0 (three successive symbols are equal), R1 (two successive symbols are equal and the remaining one symbol is different), LR2 (three successive symbols are in ascending or descending order), UR2 (second symbol is larger or smaller compared to the first and third symbol) and ALT (three successive symbols are alternating between “+1" and “ -1 "). Subsequently, the percentage of occurrence of each word type was determined by dividing the total count of a particular word pattern divided by the total count of all words.

For the analysis of effect of respiratory inhalationexhalation phase transition on low-high alterations in HRV symbolic dynamics, we calculated ALT: (a) including all the word types with alternating symbolic sequences, and (b) same as (a) but excluding the words that occur during respiratory phase transitions.

\subsection{Statistical analysis}


GraphPad Prism version 6.05 for Windows (GraphPad Software, San Diego, California, USA) was used for statistical analysis. HRV symbolic parameters were logtransformed to achieve normal distribution of data. Bradley-Blackwood test (which simultaneously compares the means and variances of two measurements), Lin's concordance correlation coefficient and Bland-Altman analysis [10] was used for the assessment of reproducibility of HRV parameters measured on random 10 -second segments.

\section{Results}

Mean height of the study population was $169.6 \pm 10.4 \mathrm{~cm}$, mean weight was $69.8 \pm 15.2 \mathrm{~kg}$, and BMI was $24.2 \pm 4.6$ $\mathrm{cm} / \mathrm{kg}^{2}$. Two participants (1.2\%) were on beta-blockers. The average number of beats analyzed in both the 10second intervals was $11.25 \pm 1.79$ (Tables $1 \& 2$ ).

\subsection{Reproducibility of traditional HRV measures}

The RR' interval changes (meanNN) showed a very high reproducibility when comparing two random 10second ECG segments (Table 1). The sdNN, rMSSD and Poincare's PPA_S1S2 also showed high reproducibility but with a wider range of 95\% limits of agreement (Table 1 ). The Lin's concordance coefficient was $>0.75$ for all the variables.

Table 1. Reproducibility agreement of two 10-second ECG HRV characteristic measures $(n=170)$.

\begin{tabular}{|c|c|c|c|c|c|}
\hline Parameter & $\begin{array}{l}\text { ECG } \\
10 \text { s \#1 }\end{array}$ & $\begin{array}{l}\text { ECG } \\
10 s \# 2\end{array}$ & Bias & $\begin{array}{l}95 \% \\
\text { limits of } \\
\text { agreement }\end{array}$ & $\begin{array}{l}\text { Bradley- } \\
\text { Blackwood } \\
\text { F (P) }\end{array}$ \\
\hline Beats, $n$ & $\begin{array}{l}11.25 \\
\pm 1.79 \\
\end{array}$ & $\begin{array}{l}11.25 \\
\pm 1.79 \\
\end{array}$ & 0.000 & $\begin{array}{l}{[-1.210 ;} \\
1.210]\end{array}$ & $0.003(0.99)$ \\
\hline $\begin{array}{l}\text { meanNN, } \\
\text { ms }\end{array}$ & $\begin{array}{l}944.2 \\
\pm 152.9\end{array}$ & $\begin{array}{l}946.4 \\
\pm 155.9\end{array}$ & -2.17 & $\begin{array}{l}\text { [-80.08; } \\
75.75]\end{array}$ & $0.76(0.47)$ \\
\hline $\begin{array}{l}\text { Log } \\
\text { sdNN }\end{array}$ & $\begin{array}{l}1.394 \\
\pm 0.284 \\
\end{array}$ & $\begin{array}{l}1.378 \\
\pm 0.274 \\
\end{array}$ & 0.017 & $\begin{array}{l}-0.355 ; \\
0.388]\end{array}$ & $0.90(0.41)$ \\
\hline $\begin{array}{l}\text { Log } \\
\text { rMMSD }\end{array}$ & $\begin{array}{l}1.457 \\
\pm 0.315 \\
\end{array}$ & $\begin{array}{l}1.442 \\
\pm 0.297 \\
\end{array}$ & 0.015 & $\begin{array}{l}{[-0.380 ;} \\
0.410]\end{array}$ & $1.17(0.31)$ \\
\hline $\begin{array}{l}\text { Log } \\
\text { PPA_S1S2 }\end{array}$ & $\begin{array}{l}-0.887 \\
\pm 0.363\end{array}$ & $\begin{array}{l}-0.904 \\
\pm 0.353\end{array}$ & 0.017 & $\begin{array}{l}{[-0.395 ;} \\
0.429]\end{array}$ & $0.74(0.48)$ \\
\hline
\end{tabular}

\subsection{Reproducibility of $\mathrm{HRV}$ indices calculated using JSD}

The HRV symbolic dynamics calculated using JSD showed low agreement between the two random 10second segments (Table 2). The Lin's concordance coefficient was $<0.3$ for all the JSD indices. The BlandAltman plot in Fig. 2 shows the limits of agreement for the JSD variable, R1, measured at two random 10-second segments.
Table 2. Reproducibility agreement of two 10-second HRV measures using JSD ( $n=170)$.

\begin{tabular}{|c|c|c|c|c|c|}
\hline Parameter & $\begin{array}{l}\text { ECG } \\
10 s \# 1\end{array}$ & $\begin{array}{l}\text { ECG } \\
10 \text { s \#2 }\end{array}$ & Bias & $\begin{array}{l}95 \% \\
\text { limits of } \\
\text { agreement }\end{array}$ & $\begin{array}{l}\text { Bradley- } \\
\text { Blackwood } \\
\text { F (P) }\end{array}$ \\
\hline Beats, $n$ & $\begin{array}{l}11.25 \\
\pm 1.79\end{array}$ & $\begin{array}{l}11.25 \\
\pm 1.79\end{array}$ & 0.000 & $\begin{array}{l}\text { [-1.210; } \\
1.210]\end{array}$ & $0.003(0.99)$ \\
\hline LogR0 & $\begin{array}{l}1.305 \\
\pm 0.224\end{array}$ & $\begin{array}{l}1.303 \\
\pm 0.210\end{array}$ & 0.047 & $\begin{array}{l}{[-0.489 ;} \\
0.583]\end{array}$ & $0.80(0.45)$ \\
\hline LogR1 & $\begin{array}{l}1.719 \\
\pm 0.199 \\
\end{array}$ & $\begin{array}{l}1.741 \\
\pm 0.174\end{array}$ & -0.020 & $\begin{array}{l}{[-0.460 ;} \\
0.420]\end{array}$ & $0.78(0.46)$ \\
\hline LogLR2 & $\begin{array}{l}1.135 \\
\pm 0.136 \\
\end{array}$ & $\begin{array}{l}1.134 \\
\pm 0.146 \\
\end{array}$ & -0.031 & $\begin{array}{l}-0.397 ; \\
0.316]\end{array}$ & $0.17(0.84)$ \\
\hline LogUR2 & $\begin{array}{l}1.502 \\
\pm 0.274 \\
\end{array}$ & $\begin{array}{l}1.468 \\
\pm 0.253\end{array}$ & 0.025 & $\begin{array}{l}{[-0.594 ;} \\
0.644]\end{array}$ & $1.09(0.34)$ \\
\hline LogALT & $\begin{array}{l}1.468 \\
\pm 0.291 \\
\end{array}$ & $\begin{array}{l}1.431 \\
\pm 0.253 \\
\end{array}$ & 0.024 & $\begin{array}{l}{[-0.612 ;} \\
0.661]\end{array}$ & $0.57(0.57)$ \\
\hline
\end{tabular}

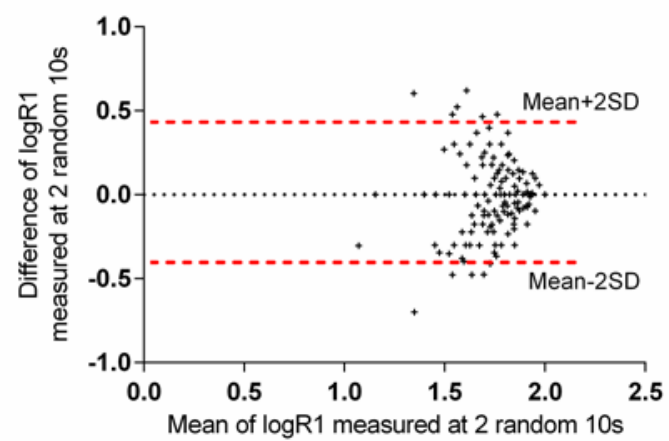

Figure 2. Bland-Altman plot demonstrating agreement of log-transformed symbolic R1 (logR1) at 2 random 10second segments.

\subsection{Effect of respiratory phase changes on HRV symbolic characteristics}

Percentage of low-high alterations in HRV symbolic dynamics (ALT) after excluding the words that occur during respiratory phase transitions showed a significant decrease as compared to ALT calculated without taking respiratory phases into account (10s: $4.7 \pm 9.4$ vs. $24.8 \pm 21.0 \%, p<0.0001$; 3min: $9.8 \pm 8.1$ vs. $24.8 \pm 12.3 \%$, $p<0.0001$, respectively) as seen in Fig.3.

\section{Discussion}

This is the first paper to investigate reproducibility of HRV characteristics using symbolic dynamic approach measured on 10-second of ECG. Our results indicate that while traditional HRV measures such as meanNN, Poincare plot show high reproducibility, limits of agreement of HRV parameters calculated using JSD are wider. In addition, the alternans phenomenon of HRV symbolic dynamics is associated with the respiratory phase transitions. 

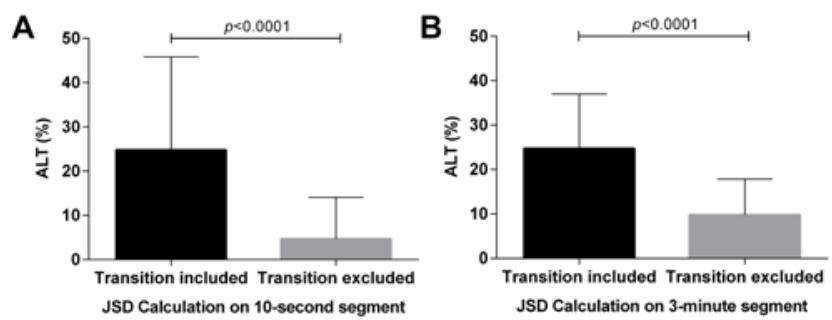

Figure 3. Comparison of percentage of HRV alternans (ALT) calculated using JSD on, A: 10-second segments and B: 3-minute segments, including and excluding respiratory phase transitions.

Robust reproducibility of a method is necessary for its clinical use and appropriate interpretation of results. In this study, although we observed high agreement of traditional HRV characteristic measures, the HRV symbolic measures using JSD showed low agreement between two random 10-second recordings. However, the number of beats analysed in both the 10-second recordings were highly correlated in each subject, thus implying similar heart rate for both the segments. This suggests that a longer than 10 -second ECG recording would be more appropriate. However, the 10-second ECG is widely available and utilizing the JSD approach could help characterize the phenomenon of HRV dynamics. In addition, a 10-second recording is considered to be stationary and hence is less likely to be affected by factors such as noise compared to a longer recording. Further investigations are needed to find the best JSD approach.

Beat-to-beat variations within a repetitive alternation of measurement parameters such as heart rate are considered to be important in the functional assessments of patients. The quantification of HRV for predefined sections of the respiratory cycle improves risk stratification of post-myocardial infarction (MI) death. Bivariate phase-rectified signal averaging method showed a strong association between expiration-triggered shortening of RR intervals and death after MI [11]. In this study we observed a significant decrease in low-high alterations of HRV symbolic dynamics after exclusion of respiratory phase transitions in both 10 -second and the whole 3-minute recordings. This suggests that alterations in HRV occurring during respiratory phase transitions must be excluded to study the true phenomenon of HRV symbolic dynamics. Further clinical studies are required to understand the phenomenon of HRV symbolic dynamics and their clinical implications.

\section{Conclusion}

The HRV parameters calculated using JSD have low reproducibility on 10-second ECG. Respiration-induced ECG changes should be considered for the study of HRV symbolic dynamics.

\section{Acknowledgements}

The authors thank the participants and staff who contributed to the IDEAL study. This work was supported by the National Institute of Health \#1R01HL118277.

\section{References}

[1] Lombardi F. Clinical implications of present physiological understanding of HRV components. Card Electrophysiol Rev 2002;6:245-9.

[2] Baumert M, Baier V, Truebner S, Schirdewan A, Voss A. Short- and long-term joint symbolic dynamics of heart rate and blood pressure in dilated cardiomyopathy. IEEE Trans Biomed Eng 2005;52:2112-5.

[3] Kabir MM, Saint DA, Nalivaiko E, Abbott D, Voss A, Baumert M. Quantification of cardiorespiratory interactions based on joint symbolic dynamics. Ann Biomed Eng 2011;39:2604-14.

[4] Caminal P, Vallverdu M, Giraldo B, Benito S, Vazquez G, Voss A. Optimized symbolic dynamics approach for the analysis of the respiratory pattern. IEEE Trans Biomed Eng 2005;52:1832-9.

[5] Brisinda D, Venuti A, Cataldi C, Efremov K, Intorno E, Fenici R. Real-time imaging of stress-induced cardiac autonomic adaption during realistic force-on-force police scenarios. J Police Crim Psych 2015;30:71-86.

[6] Baumert M, Kabir MM, Dalouk K, Henrikson CA, Tereshchenko LG. VV' alternans triplets on near-field ICD intracardiac electrogram is associated with mortality. Pacing Clin Electrophysiol 2015;38:547-57.

[7] Couderc JP. The telemetric and Holter ECG warehouse initiative (THEW): A data repository for the design, implementation and validation of ECG-related technologies. Conf Proc IEEE Eng Med Biol Soc 2010;2010:6252-5.

[8] Lazaro J, Alcaine A, Romero D, Gil E, Laguna P, Pueyo E, Bailon R. Electrocardiogram derived respiratory rate from QRS slopes and R-wave angle. Ann Biomed Eng 2014;40:2072-83.

[9] Porta A, Guzzetti S, Montano N, Furlan R, Pagani M, Malliani A, Cerutti S. Entropy, entropy rate, and pattern classification as tools to typify complexity in short heart period variability series. IEEE Trans Biomed Eng 2001;48:1282-91.

[10] Bland JM, Altman DG. Statistical methods for assessing agreement between two methods of clinical measurement. Lancet 1986;1:307-10.

[11] Bauer A, Morley-Davies A, Barthel P, Muller A, Ulm K, Malik M, Schmidt G. Bivariate phase-rectified signal averaging for assessment of spontaneous baroreflex sensitivity: pilot study of the technology. J Electrocardiol 2010;43:649-53.

Address for correspondence.

Muammar M. Kabir

Knight Cardiovascular Institute,

Oregon Health and Science University, Portland, OR 97239, USA

Email: kabir@ohsu.edu 